Volume 8, No. 7, July - August 2017

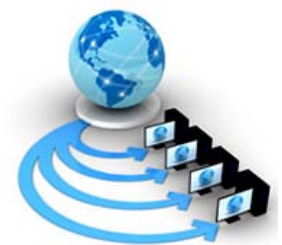

International Journal of Advanced Research in Computer Science

RESEARCH PAPER

\author{
Available Online at www.ijarcs.info
}

\title{
FLOOR PLANNING DESIGN USING k-NEAREST NEIGHBOUR BASED BIAS TRANSFERS LEARNING ALGORITHM
}

\author{
Utkarsh Kumar Singh \\ School of information \& technology (SoICT) VLSI Design \\ Gautam Buddha University \\ Greater Noida (U.P) India
}

\author{
Preeti Singh (Lecturer) \\ Department of Electronics \\ Government Polytechnic College \\ Pilibhit (U.P) India
}

\author{
Himanshu Kumar* \\ School of information \& technology (SoICT) Computer Science \\ Gautam Buddha University \\ Greater Noida (U.P) India
}

\begin{abstract}
In VLSI chip the minimization of wire length is most significant, its play a very important role for various application. The aim of this work is to minimize the wire length through finding an optimum resolution for VLSI physical project mechanisms like dividing and floorplanning. In very large-scale integration circuit splitting, the issue of finding a smallest delay has prime significance. The very large-scale integration circuit floor planning, the issue of reducing silicon region is also a crucial issue. Decreasing the delay in dividing area here floor planning helps to reduce the wire-length. In our research Bias Transfer (BITER) is an Evolutionary Algorithm that suitable for transfer learning in agents in floor planning phases within its evolutionary cycle to obtain the minimum wire length and make resize then in needed instead of the MA uses the for exploration and uses the local search method for exploitation and its comparison between the GA and MA is to justify the effectiveness of the Bias Transfer-KNN Technique which reducing delay and dead space area at the time of in partitioning that simulated in MATLAB 2014Ra.
\end{abstract}

Keywords: Bias Transfer, floor plan, VLSI, MA, GA, KNN etc.

\section{INTRODUCTION}

Floor planning is that the terribly central stage in VLSI physical style for class conscious structure component style method. Floor planning affords initial response which estimates architectural choices, approximation of chip space, estimations delay; interconnect length and congestion due to wrong wiring[2]. Such as knowledge improvements, style complexness is increasing and therefore the circuit scope is obtaining larger. Thus space of the circuit gets increase and tougher to minimizing the interconnect length. The VLSI floor planning is that the NP onerous downside[3]. So it's horribly troublesome to seek out the best solution. During this paper we tend to take into account, a Bias Transfer (BITER) program primarily based floor planning has been developed with novel rule to transfer learning in VLSI system.

The duration "transfer learning” is a properly refined time for approximately that can be measured an essential module of any knowledge energy of a mortal or object: the base explanation to a novel issue on capability and knowledge achievement of former learning tasks[1]. It is approximately which a wisdom entity does obliquely since genetic on: no job always inaccessible then implanted in a mutual close or past. For this lifelong learning contrary type background, In Reinforce Learning transfer [6],[7] which accepts two altered $\mathrm{M}$ and $\mathrm{M}$ ' which have approximately" in common". The unity is maximum probable assumed in an assignment plotting purpose which plots positions and movements as of $M$ to $M$ ' such as center for recycling knowledgeable rules. The job maps the assumed through mortal administrators or knowledgeable, and then frequently here is specific example effective the wisdom agent which advantage from its involvement. The precise mutual disputes: Now it is assignment $M$, there is job M', and exactly how it can bridge among them. It is an equally constricted opinion on indication reuse. Novel organic and independent variations of information transmission are required.

\section{PROBLEM STATEMENT}

Problem of floor planning will be specified as monitors: suppose $\mathrm{m}=\{\mathrm{m} 1, \mathrm{~m} 2, \ldots, \mathrm{mm}\}$ be situated a established of $\mathrm{m}$ mechanisms of rectangular who's aim for particular (height, width and part are signified through wi, hi, and ai, $1 \leq \mathrm{i} \leq \mathrm{n}$. Every component is free to substitute. Supposing (xi, yi) signify and establish the bottom-left angle of module $1 \leq \mathrm{i} \leq \mathrm{n}$, on a chip. A floorplan $\mathrm{f}$ is a job of (xi, yi) for each bi, $1 \leq \mathrm{i} \leq \mathrm{m}$ like that no two unit's association with each former. The aim of floor planning is to improve an already defined cost metric which are group of the capacity (such as the least bounding square of $\mathrm{f}$ ) and wire length (i.e., the amount of all interconnection lengths) prompted through a floorplan. To present floorplan strategies, new costs like as routability, influence, and current strength similarly requirement to be measured. Ease of use.

\section{SYSTEM MODEL}

In SVM the kernels are used to plan the non-linear involved information interested in an advanced dimensional character 
space whenever the computational device of the linear knowledge device is amplified [10],[12],[13]. By the kernel purpose, the optimization organization resolve in the high measurement can be assumed as:

$$
\mathrm{f}(\mathrm{x})=\operatorname{sgn}\left(\sum_{\mathrm{i}=1}^{\mathrm{Ns}} \alpha_{\mathrm{i}} \mathrm{y}_{\mathrm{i}} \mathrm{K}\left(\overrightarrow{\mathrm{x}}_{\mathrm{i}}, \overrightarrow{\mathrm{x}}\right)+\mathrm{b}\right.
$$

Currently, $\mathbb{K}\left(\overrightarrow{\mathrm{x}}_{\mathrm{i}}, \overrightarrow{\mathrm{x}}_{\mathrm{j}}\right)$ is used as kernel determination in SVM. It dealings the parallel or distance between the two vectors. A kernel properties $\mathrm{K}: \mathrm{X} \times \mathrm{X} \rightarrow \mathrm{R}$ in $\mathrm{K}$ is actual if there is certain feature plotting $\Phi$, such that

$$
\mathrm{K}\left(\overrightarrow{\mathrm{x}}_{\mathrm{i}}, \overrightarrow{\mathrm{x}}_{\mathrm{j}}\right)=\Phi\left(\overrightarrow{\mathrm{x}}_{\mathrm{i}}\right) \cdot \Phi\left(\overrightarrow{\mathrm{x}}_{\mathrm{j}}\right)
$$

Therefore, we can compute $\left(\Phi\left(\overrightarrow{\mathrm{x}}_{\mathrm{i}}\right), \Phi\left(\overrightarrow{\mathrm{x}}_{\mathrm{j}}\right)\right)$ as dot product without cooperatively connecting function $\Phi$ to response vector. The kernel job can alter the dot invention processes in in elevation length interplanetary into the kernel function processes in input space as extensive as it fulfills the Mercer complaint [5], [11], [14]; thus it ignores the issues of direct computation in high length space and resolves the length adversity.

The presentation of SVM mostly determined arranged the kernel purpose. Each function of kernel has its individual benefits and losses. Numerous likelihoods of kernels occur and it is problematic to clarify their separate types. In particular circumstance single kernel function good capacity is not good as well as generalization ability.

Support vector machine has two kinds of Kernel purposes of: local kernel purpose and worldwide kernel purpose. Working of worldwide kernel purpose as samples far away as each other which have switched on the worth of kernel purpose; But in local kernel it only closed samples to each other that impact on the value of kernel function[4]. The Polynomial kernel is an instance of worldwide kernel purpose and Radial Basis Polynomial kernel is an instance of limited kernel function.

\section{A. RBF kernel}

In function of Radial Basis Polynomial kernel, it is most generally used in kernel purpose due to its best learning capacity between each single kernel purposes.

$$
\mathrm{k}\left(\overrightarrow{\mathrm{x}}_{\mathrm{i}}, \overrightarrow{\mathrm{x}}_{\mathrm{j}}\right)=\mathrm{e}^{-\left\|\overrightarrow{\mathrm{x}}_{\mathrm{i}}-\overrightarrow{\mathrm{x}}_{\mathrm{j}}\right\|^{2} / 2 \sigma^{2}}
$$

where, ${ }^{\sigma^{2}}=$ mean $\left\|\vec{x}_{i}-\vec{x}_{j}\right\|^{2}$

The radial basis function kernel will easily modify in numerous circumstances, low-dimension, high-dimension, minor example, huge example, etc. Radial Basis Polynomial has the benefit of taking less parameter. A big no. of arithmetical investigates showed which are the ability of learning then RBF is contrariwise relative to the consideration $\sigma[14] . \sigma$ regulates the part of effect done the data space.

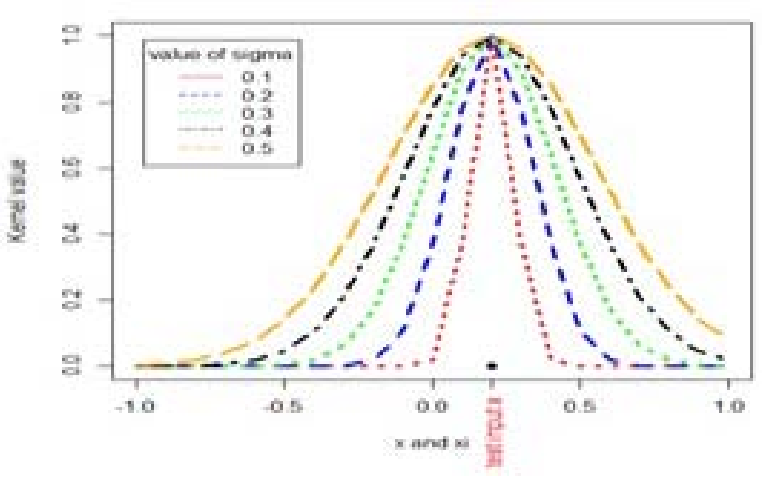

Figure 1. A narrow RBF kernel purpose with altered value of $\sigma$

A superior worth of $\sigma$ resolve provide a level result apparent and further systematic resolution margin. Its aim behind a Radial Basis Polynomial through big $\sigma$ allows a SVM has robust incentive done a huge zone. If $\sigma$ is precise size which shown fig. 1 which only instances which length is near to $\sigma$ can be precious. Subsequently, it results on the information facts in the locality of the investigation opinion, it can be call as narrow kernel.

\section{B. Polynomial kernel}

The polynomial kernel purpose is determining as

$$
\mathrm{k}\left(\overrightarrow{\mathrm{x}}_{\mathrm{i}}, \overrightarrow{\mathrm{x}}_{\mathrm{j}}\right)=\left(\overrightarrow{\mathrm{x}}_{\mathrm{i}} \cdot \overrightarrow{\mathrm{x}}_{\mathrm{j}}+1\right)^{\mathrm{d}}
$$

Wherever $\mathrm{d}$ is the grade of the kernel.

The polynomial kernel is a worldwide kernel purpose with best generalization capacity that can mark the worth of worldwide kernel, however deprived of the robust learning capability like local kernel purpose RBF.

\section{Kernel function for learning}

In the support vector machine classifier, it selects precise kernel purpose resources selecting a method of plotting to project the input planetary into a feature space. A knowledge ideal, which is mediated through its learning capacity and forecast capacity, was fabricated up through indicating a precise kernel function. Therefore, to construct up a prototypical which has good knowledge as well as good forecast ability; this research has mutual the benefits of both, local RBF kernel function and overall Polynomial kernel purpose.

A new function of kernel called Radial Basis Polynomial Kernel (RBPK) is currently well-defined as:

$$
\mathrm{k}\left(\mathrm{x}_{\mathrm{i},}, \mathrm{x}_{\mathrm{j}}\right)=\exp \left(\frac{\left(\mathrm{x}_{\mathrm{i}}, \mathrm{x}_{\mathrm{j}}+\mathrm{c}\right)^{\mathrm{d}}}{\sigma^{2}}\right)
$$

\section{Where $\mathrm{c}>0$ and $\mathrm{d}>0$}

This RBPK kernel function proceeds benefit of best forecast capability after polynomial kernel and good knowledge capability from RBF kernel determination.

The Mercer's proposition gives the essential and necessary ailment for an effective kernel purpose. It states that a kernel purpose is an allowable kernel if the equivalent kernel environment is symmetric and optimistic semi-definite [9], [8], [5]. Subsequently, the RBPK kernel fulfils the Mercer's formula it is an allowable kernel. 


\section{PROPOSED TECHNIQUE FOR FLOOR PLANNING}

\section{A. BITER-KNN (bias transfer $k$ nearest neighbor)}

To create Rule Bias Transfer operative who treat the interpreted judgment list as data slightly as rules:

a. The advantage of agent since the decision lists firstly, but it refines plan as it collects additional knowledge in the object task.

b. The learner gives this pseudo-action as a regular accomplishment.

c. Toward the bias learner to this achievement, the manager is compulsory to perform the pseudo act for a perpetual no. of incidents at the establishment of training in the objective task.

d. Supposing negative initialization, the pseudo-action can have industrialized Q-values than all added movements, that reason the mediator to initially make possible activities, but over time learning can dominate this bias.

e. Now add Extra Variable for an extra state mutable to the object task's state explanation.

f. The KNN procedure is the technique in ML which classified through a mainstream selection of its neighbors, with the determination assigned occurrence for most mutual class amongst its $\mathrm{k}$ nearest neighbors

g. Classically Euclidean distance is used as the distance metric; however, this is only suitable to endless variables and finds dead space area.

h. In classification of text, alternative metric, intersection metric or Hamming distance can be reducing size of betweeness of modules.

i. The adaptable data precedes the worth of directory for the accomplishment recommended concluded Dt. To support the agent in knowledge the position of this variable, we once more originally force the mediator to select the act recommended by Dt.

j. Currently it acquires the significance of this state adaptable, and then it can still acquire to ignore the formal variable once the advice is sub-optimal.

\section{B. Rule Transfer procedure:}

The given stages précis Rule Transfer:

1. Obtain a rule $(\pi: S \rightarrow A)$ in the basis of given task. Later training has completed, or during the concluding training segment the agent proceedings some no. of connections with the condition in the mode of $(S, A)$ pairs while given the learned policy.

2. Now it take a decision list (Ds: $\mathrm{S} \rightarrow \mathrm{A}$ ) which précises the source strategy. Subsequently the information is collected; a rule learner that used to condense the composed data to estimate the learned policy.

3. Improve the resolution list for use in the object task (Decode (Ds) $\rightarrow$ Dt). To permit the well-read assessment list to be useful in a target job that has dissimilar state variables and activities from the basis task, the decision list necessity be transformed before it can be used.

4. Procedure Dt to absorb a strategy in the objective task. Segment 4 will deliberate how the transmitted rules will help in the objective job to speed up knowledge.
The main modification among this allocation technique and foregoing work is which we influence rules to give rules as rule learning and fast and well assumed, and they are mortal legible.

\section{Precise Steps for our proposed Approach:}

1. Assign local search to floor planning

2. check initial distance and its array //BSF/DSF make tree structure KDD

3. To check local search using KNN

4. Now calculate hamming distance to each module.

5. find next block connectivity using KNN

6. Continuous no. of occurrences at the opening of training in the mark task for same hamming distance.

7. find generation on the basis of local search

8. First update and store kernel space used for reducing dead space using learning transfer to assign individual layer.

\section{RESULT}

The floor planning Representation, Bias learning transfer Process Operation and Determined Area Design is approved out in MATLAB programming language.

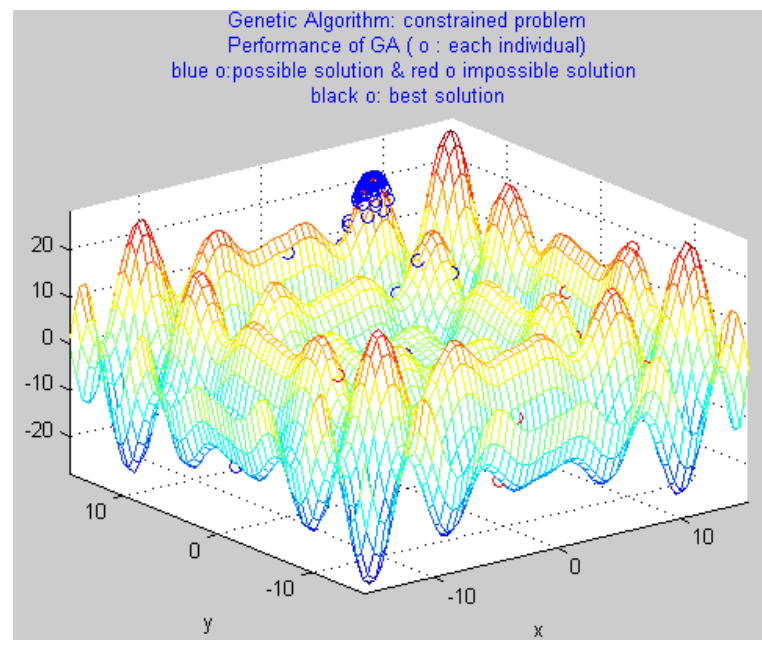

Figure 2. A scenario of possible floorplanning

Above figure represent the possible, impossible and best solution for floor planning, here blue area shows the possible solution, red area show the impossible area and circular area shows best solution.

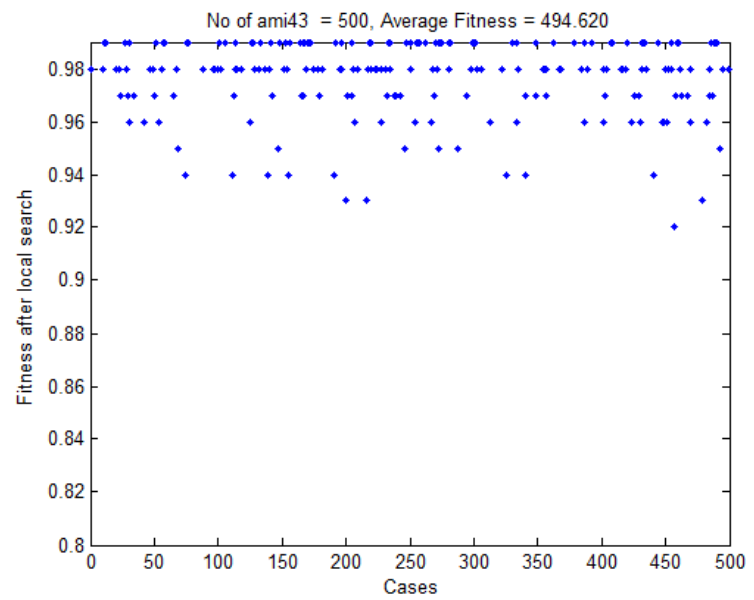

Figure 3. Fitness value after local search

In this figure we get no of ami43 $=500$ and fitness $=494.620$ after local search using KNN method. 
In above figure the value of fitness is creating on the quantity of dead space formed in every floorplan definite through the separate standardized postfix terms programmed in the population as presented in figure 4 and 5. The main parent is designated deterministically in classification, but the second parental is designated in a roulette wheel mode, the selection likelihoods for every genotype creature designed using the subsequent formulation:

\section{Selection probability $=($ Rank $) / \sum$ Pranks}

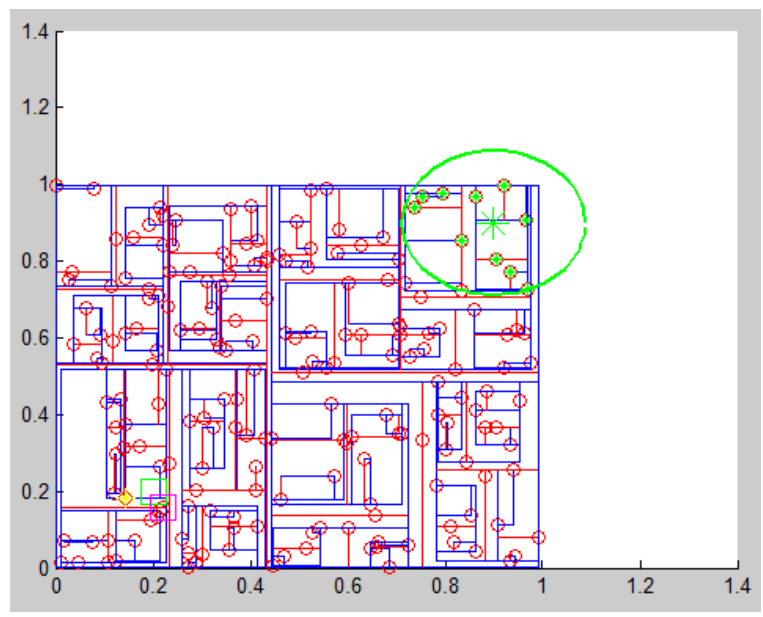

Figure 4. Floorplan using Bias transform

The outcomes of our proposed method are précised in all figures. We procedure of the components from the standard information groups ami43. Column 1 provides the outline tractability of the simple squares for the experimentations in the rows and columns 3 to 4 précis the dead space achieved through the genetic algorithm. The measurement of dead space for the best floorplan is verified over five reproduce shots of the Genetic algorithm in pole 3, and the whole best for from the five runs is exposed in column 5 . The total no. of postfix appearance assessments are around over the five replicate runs is well-known in column 4 , and the typical run time looks in pole 5 .

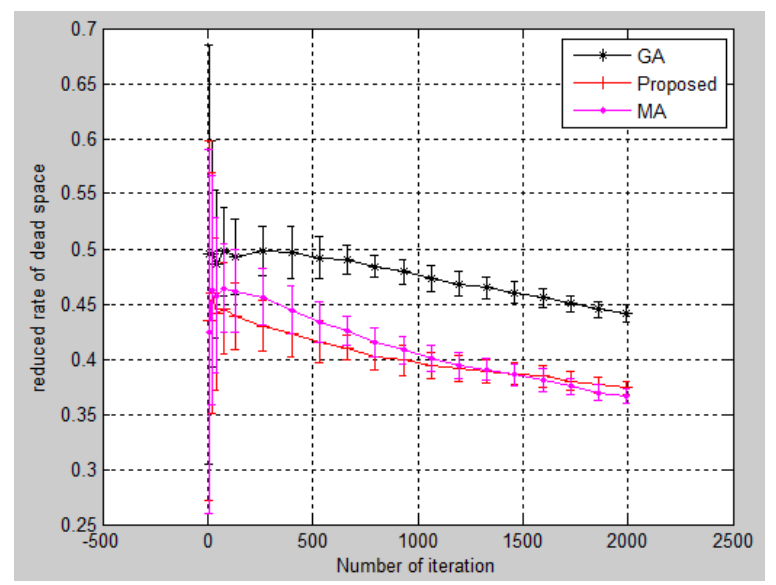

Figure 5. reduced rate of dead space

In figure 4 the proposed method get low dead space occurrence.

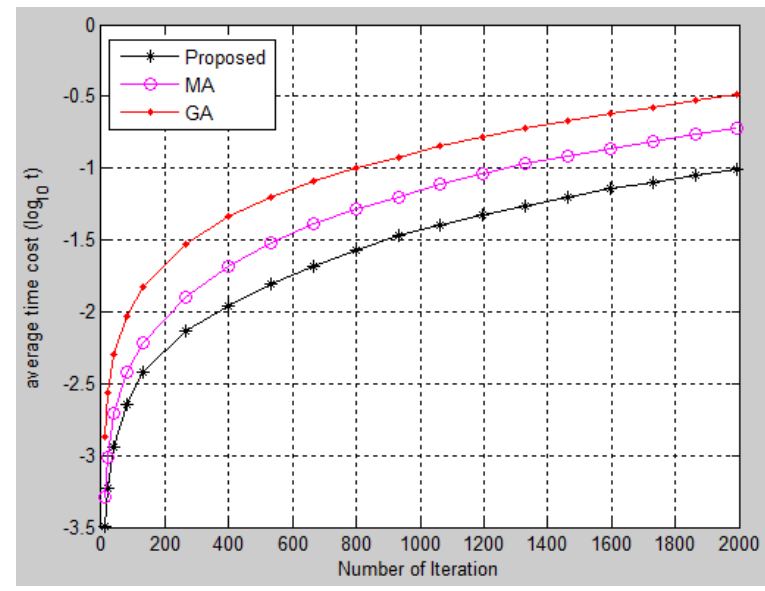

Figure 6. Average time cost on iteration

Here genotypes are ordered permitting to the values of the dead space which created with the worst categorized 1, the 2nd worst 2 etc. and the best categorized highest. The genetic algorithm is types of permutations of archives from decoder create and regularized postfix strings. These strings are, in turn, managed by a stack to produce a floorplan.

In figure 5 we can see that the average time cost of proposed work is lower than the MA and GA.

Table I. Proposed Technique table results parameter

\begin{tabular}{|c|c|c|c|c|}
\hline $\begin{array}{c}\text { Algorithm } \\
\text { Used }\end{array}$ & Time & Std & mean & $\begin{array}{c}\text { Reduced } \\
\text { Dead } \\
\text { Space }\end{array}$ \\
\hline GA: & 38.6775 & 0.6463 & $\begin{array}{c}3372.500 \\
0\end{array}$ & 881 \\
\hline MA: & 37.3800 & 0.5921 & $\begin{array}{c}4944.800 \\
0\end{array}$ & 760 \\
\hline $\begin{array}{c}\text { Proposed } \\
:\end{array}$ & 36.6250 & 0.6142 & $\begin{array}{c}5011.000 \\
0\end{array}$ & 720 \\
\hline
\end{tabular}

\section{CONCLUSION}

The bias-learning transfer process for present Floor planning issues through Fixed-outline is scheduled the new MA and genetic algorithm. The Investigational performance have presented that proposed method (bias-learning) indicates to nearer and constant merging to anticipated Floorplan explanations that is more actual as associate to memetic algorithm (MA). To fixed-outline floor planning, when novel cost function seeing the proposed technique more capably to find floor plans inside the assumed chip outline as associate to MA and GA. The investigational outcomes on the constant outline on Floorplan which have presented the effectiveness and efficiency to reduce dead space area and its mistakes of our Floor planning procedures; for those uses, our outcomes outstrip the connected modern study through large margins.

\section{REFERENCES}

[1] Singh, S. P., \& Sutton, R. S. (1996). Reinforcement learning with replaceing eligibility traces. Machine Learning, 22, 123158. 
[2] A. B. Kahng, "Classical floorplanning harmful?,” in Proc. Int. Symp.Phys. Design, 2000, pp. 207-213.

[3] R. H. J. M. Otten, "Efficient floorplan optimization," in Proc. Int. Conf.Comput. Design, 1983, pp. 499-502.

[4] S. N. Adya and I. L. Markov, "Fixed-outline floorplanning through better local search,” in Proc. Int. Conf. Comput. Design, 2001, pp. 328-334.

[5] Campbell, Colin, and Y. Ying, "Learning with support vector machines."Synthesis Lectures on Artificial Intelligence and Machine Learning vol. 5, no. 1: 1-95, 2011.

[6] Matthew E Taylor and Peter Stone. Transfer learning for reinforcement learning domains: A survey. The Journal of Machine Learning Research, 10:1633-1685, 2009.

[7] Cortes, Corinna, and V. Vapnik. "Support-vector networks" Machine learning vol. 20, no. 3: 273-297, 1995.

[8] Maolin Tang, Raymond Y. K. Lau," A Parallel Genetic Algorithm for Floorplan Area Optimization", Seventh International Conference on Intelligent Systems Design and Applications,IEEE, 2007, pp. 801-806
[9] Guolong Chen, Wenzhong Guo, Yuzhong Chen, "A PSO-based intelligent decision algorithm for VLSI Floorplanning", Soft Computing, VOL. 14, NO 12, Springer, 2009, pp. 1329-1337

[10] Han, Jiawei, and M. Kambe,” Data Mining, Southeast Asia Edition: Concepts and Techniques”. Morgan kaufmann, 2006.

[11] Herbrich, Ralf. "Learning Kernel classifiers: theory and algorithms (adaptive computation and machine learning)." MIT press, 2001

[12] Mu, Xiangyang, and Y. Zhou. "A Novel Gaussian Kernel Function for Minimax Probability Machine." In Intelligent Systems, 2009. GCIS'09. WRI Global Congress on, vol. 3, pp. 491-494. IEEE, 2009.

[13] Muller, K., S. Mika, G. Ratsch, Koji Tsuda, and Bernhard Scholkopf. "An introduction to kernel-based learning algorithms." IEEE Transactions on Neural Networks, 12, no. 2: 181-201, 2001.

[14] Schölkopf, Bernhard, and A. J. Smola, "Learning with kernels: support vector machines, regularization, optimization, and beyond”. MIT press, 2002. 